\title{
TITLE:
}

\section{A branch-and-bound method for absolute value programs}

\author{
$\operatorname{AUTHOR}(S)$ :
}

Yamanaka, Shota; Fukushima, Masao

\section{CITATION:}

Yamanaka, Shota ...[et al]. A branch-and-bound method for absolute value programs. Optimization 2011, 63(2): 305-319

\section{ISSUE DATE:}

2011-11-10

URL:

http://hdl.handle.net/2433/196860

\section{RIGHT:}

This is an Accepted Manuscript of an article published by Taylor \& Francis in Optimization on 2011, available online: http://www.tandfonline.com/10.1080/02331934.2011.644289; This is not the published version. Please cite only the published version.; この論文は出版 社版でありません。引用の際には出版社版をご確認ご利用ください。 
Optimization

Vol. 00, No. 00, Jul 2011, 1-13

\title{
RESEARCH ARTICLE
}

\section{A Branch-and-Bound Method for Absolute Value Programs}

\author{
Shota Yamanaka ${ }^{a}$ and Masao Fukushima ${ }^{a *}$ \\ ${ }^{a}$ Department of Applied Mathematics and Physics, Graduate School of Informatics, \\ Kyoto University, Kyoto 606-8501, Japan
}

(August 2011)

\begin{abstract}
In recent years, the absolute value equation (AVE) has attracted a growing attention. The absolute value program (AVP) is an extension of AVE, which contains absolute values of variables in its objective function and constraints. In this paper, we propose an algorithm for the AVP, which is based on the branch-and-bound method. In the branching procedure, we generate two subproblems by restricting the sign of a variable to be nonnegative or nonpositive. In the bounding procedure, we utilize the duality results for AVP. Furthermore, we carry out numerical experiments for nonconvex multi-facility location problems to show the validity of the proposed algorithm.
\end{abstract}

Keywords: Absolute value program, absolute value equation, branch-and-bound method, nonconvex facility location problem

AMS Subject Classification: 90C30, 65K05, 90C57

\section{Introduction}

In recent years, the absolute value equation (AVE) $[1,4,9,11,12,14-16,20]$ has attracted a growing attention. The absolute value program (AVP) is an extension of AVE, which contains absolute values of variables in its objective function and constraints. Formally, the AVP is stated as follows:

$$
\begin{aligned}
(\mathrm{P}) \quad \min & c^{\top} x+d^{\top}|x| \\
\text { s.t. } & A x+B|x|=b, \\
& H x+K|x| \geq p,
\end{aligned}
$$

where $c, d \in \mathbb{R}^{n}, b \in \mathbb{R}^{m}, p \in \mathbb{R}^{\ell}, A, B \in \mathbb{R}^{m \times n}, H, K \in \mathbb{R}^{\ell \times n}$, and $|x|$ denotes the vector $|x|=\left(\left|x_{1}\right|,\left|x_{2}\right|, \ldots,\left|x_{n}\right|\right)^{\top} \in \mathbb{R}^{n}$. Although this problem is a nonconvex optimization problem in general, Mangasarian [10] showed an interesting weak duality result and a sufficient optimality condition for the problem. In addition, the AVE that appears in the constraints of the AVP is shown to be equivalent to a linear complementarity problem $[10,14]$. This result indicates that the AVP is equivalent to a linear program with linear complementarity constraints, which is a special case of the mathematical program with equilibrium constraints (MPEC) [7]. MPEC has many applications in various areas such as economics, engineering,

*Corresponding author. Email: fuku@i.kyoto-u.ac.jp 
and transportation. However, MPEC is in general difficult to deal with, since its feasible region is necessarily nonconvex and even disconnected. The study on AVP is in its infancy and, to the authors' knowledge, there have been no work except for the above-mentioned duality results of Mangasarian [10].

In this paper, we first propose an algorithm for the AVP, which is based on the branch-and-bound method. In the branching procedure, we generate two subproblems by restricting the sign of a component of the variable $x$ in $(\mathrm{P})$ to be nonnegative or nonpositive. In the bounding procedure, we utilize the duality results in AVP to obtain a lower bound for each subproblem. Furthermore, to examine the effectiveness of the proposed algorithm, we apply it to solve facility location problems (FLPs). By using the $\ell_{1}$ norm as a distance function, an FLP can naturally be formulated as an AVP. In particular, we can use the AVP formulation to deal with a nonconvex region in which facilities are located. We stress that such a problem is considerably difficult to solve compared with the conventional FLPs that assume the convexity of the region.

The paper is organized as follows. In the next section, we give some preliminary results about the AVP. Using these results, we propose a branch-and-bound algorithm for solving AVPs in Section 3. In Section 4, we carry out some numerical experiments with the proposed algorithm for two types of nonconvex FLPs. Finally, we conclude the paper in Section 5.

\section{Duality}

The dual problem of AVP (P) is defined as follows [10]:

$$
\begin{aligned}
\max & b^{\top} u+p^{\top} v \\
\text { s.t. } & \left|A^{\top} u+H^{\top} v-c\right|+B^{\top} u+K^{\top} v \leq d, \\
& v \geq 0 .
\end{aligned}
$$

Note that the inequality constraint can be represented as

$$
\begin{aligned}
& \left|A^{\top} u+H^{\top} v-c\right| \leq d-B^{\top} u-K^{\top} v \\
& \Longleftrightarrow \quad-d+B^{\top} u+K^{\top} v \leq A^{\top} u+H^{\top} v-c \leq d-B^{\top} u-K^{\top} v \\
& \Longleftrightarrow \quad\left\{\begin{array}{l}
(-A+B)^{\top} u+(-H+K)^{\top} v \leq d-c, \\
(A+B)^{\top} u+(H+K)^{\top} v \leq d+c .
\end{array}\right.
\end{aligned}
$$

Therefore, the dual problem (D) can be rewritten as follows:

$$
\begin{aligned}
\max & b^{\top} u+p^{\top} v \\
\text { s.t. } & (-A+B)^{\top} u+(-H+K)^{\top} v \leq d-c, \\
& (A+B)^{\top} u+(H+K)^{\top} v \leq d+c, \\
& v \geq 0 .
\end{aligned}
$$

Notice that the dual problem (D) is always a convex optimization problem, or more precisely, a linear program, although the primal problem $(\mathrm{P})$ is not convex in general. Moreover, a weak duality theorem and a sufficient optimality condition for AVP are shown in [10], which will be useful in our algorithm. 
Theorem 2.1: [10] If $x$ and $(u, v)$ are feasible solutions of $(\mathrm{P})$ and $(\mathrm{D})$, respectively, then the following inequality holds:

$$
c^{\top} x+d^{\top}|x| \geq b^{\top} u+p^{\top} v
$$

This theorem says that we can get a lower bound of the optimal value of $(\mathrm{P})$ by solving the dual problem (D). The next theorem gives a sufficient optimality condition for $(\mathrm{P})$.

Theorem 2.2: [10] Let $\bar{x}$ be feasible in the primal $A V P(\mathrm{P})$ and $(\bar{u}, \bar{v})$ be feasible in the dual AVP (D) with equal primal and dual objective values, that is,

$$
c^{\top} \bar{x}+d^{\top}|\bar{x}|=b^{\top} \bar{u}+p^{\top} \bar{v} .
$$

Then $\bar{x}$ and $(\bar{u}, \bar{v})$ are optimal solutions of $(\mathrm{P})$ and $(\mathrm{D})$, respectively.

\section{Branch-and-Bound Method}

In this section, we propose a branch-and-bound method for AVP. The branchand-bound method is one of fundamental global optimization methods for nonconvex optimization problems [3] and combinatorial optimization problems [5]. The method consists of branching and bounding procedures. In the branching procedure, we divide the feasible region of the original problem into some subregions to generate subproblems. On the other hand, in the bounding procedure, we check if a current subproblem can be discarded or not, by implementing some fathoming tests. We now give the detail of the branching and bounding procedures used in the proposed branch-and-bound method for solving AVPs.

A subproblem is constructed from $(\mathrm{P})$ by restricting some variables to be either nonpositive or nonnegative:

$$
\begin{aligned}
\mathrm{P}(\mathcal{I}, \mathcal{J}) \quad \min & c^{\top} x+d^{\top}|x| \\
\text { s.t. } & A x+B|x|=b, \\
& H x+K|x| \geq p, \\
& x_{i} \geq 0 \quad(i \in \mathcal{I}), \\
& x_{i} \leq 0 \quad(i \in \mathcal{J}),
\end{aligned}
$$

where $\mathcal{I}$ and $\mathcal{J}$ are subsets of $\{1,2, \ldots, n\}$ such that $\mathcal{I} \cap \mathcal{J}=\emptyset$. Note that $(\mathrm{P})=\mathrm{P}(\emptyset, \emptyset)$. The branching procedure can conveniently be explained by using the enumeration tree, where each node corresponds to a subproblem. An example of the enumeration tree with $n=2$ is shown in Fig. 1. At each node of the tree, branching means that we choose a variable $x_{i}$ and restrict it to be nonnegative or nonpositive in the corresponding subproblem. The deepest nodes in the tree correspond to $2^{n}$ linear programs, which contain no absolute values of the variables. The branch-and-bound method maintains the set of subproblems that can be selected to apply a branching procedure. Such subproblems are said to be active, and the set of the current active subproblems is denoted by $\mathcal{A}$. For example, if we generate two subproblems $\mathrm{P}(\{1\}, \emptyset)$ and $\mathrm{P}(\emptyset,\{1\})$ at the root node $\mathrm{P}(\emptyset, \emptyset)$ in the enumeration tree of Fig. 1, then we have $\mathcal{A}=\{\mathrm{P}(\{1\}, \emptyset), \mathrm{P}(\emptyset,\{1\})\}$.

In the bounding procedure, we consider the dual problem of $\mathrm{P}(\mathcal{I}, \mathcal{J})$ in order to get a lower bound of $\mathrm{P}(\mathcal{I}, \mathcal{J})$. For convenience, let $h_{i}:=\sigma_{i} e_{i} \in \mathbb{R}^{n}$ for each 


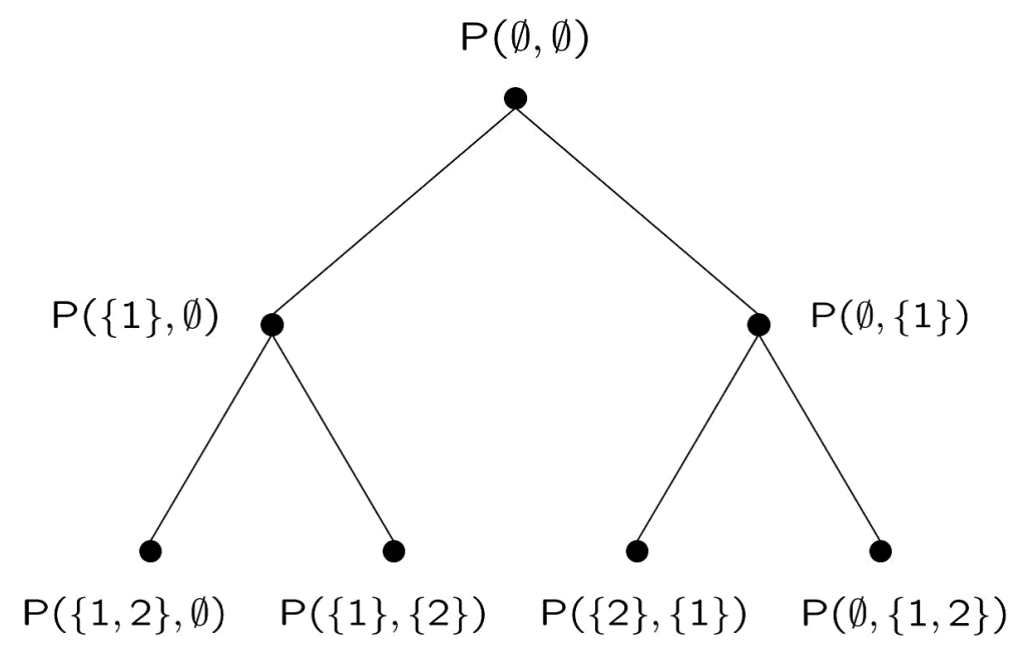

Figure 1. Enumeration tree $(n=2)$

$i \in \mathcal{I} \cup \mathcal{J}$, where $e_{i} \in \mathbb{R}^{n}$ is the $i$ th column of the $n \times n$ identity matrix, and $\sigma_{i}=1$ if $i \in \mathcal{I}$ and $\sigma_{i}=-1$ if $i \in \mathcal{J}$. Then, the nonnegativity and nonpositivity constraints on variables $x_{i}$ in $\mathrm{P}(\mathcal{I}, \mathcal{J})$ are represented as

$$
h_{i}^{T} x \geq 0 \quad(i \in \mathcal{I} \cup \mathcal{J}) .
$$

Therefore, we can rewrite $\mathrm{P}(\mathcal{I}, \mathcal{J})$ as follows:

$$
\begin{aligned}
\mathrm{P}(\mathcal{I}, \mathcal{J}) \quad \min & c^{\top} x+d^{\top}|x| \\
\text { s.t. } & A x+B|x|=b, \\
& \tilde{H} x+\tilde{K}|x| \geq \tilde{p},
\end{aligned}
$$

where $\tilde{H} \in \mathbb{R}^{(\ell+|\mathcal{I}|+|\mathcal{J}|) \times n}, \tilde{K} \in \mathbb{R}^{(\ell+|\mathcal{I}|+|\mathcal{J}|) \times n}, \tilde{p} \in \mathbb{R}^{(\ell+|\mathcal{I}|+|\mathcal{J}|)}$ are defined by

$$
\tilde{H}:=\left[\begin{array}{c}
H \\
\hline \vdots \\
h_{i}^{T} \\
\vdots
\end{array}\right], \quad \tilde{K}:=\left[\begin{array}{c}
K \\
\vdots \\
0 \\
\vdots
\end{array}\right], \quad \tilde{p}:=\left[\begin{array}{c}
p \\
\vdots \\
0 \\
\vdots
\end{array}\right]
$$

Moreover, the dual problem of $\mathrm{P}(\mathcal{I}, \mathcal{J})$ is written as

$$
\begin{aligned}
\mathrm{D}(\mathcal{I}, \mathcal{J}) \max & b^{\top} u+\tilde{p}^{\top} v \\
\text { s.t. } & \left|A^{\top} u+\tilde{H}^{\top} v-c\right|+B^{\top} u+\tilde{K}^{\top} v \leq d, \\
& v \geq 0,
\end{aligned}
$$

which can further be rewritten as a linear program. Based on the result of solving the dual problem, the subproblem $\mathrm{P}(\mathcal{I}, \mathcal{J})$ can be fathomed if one of the following conditions holds:

(i) $\mathrm{D}(\mathcal{I}, \mathcal{J})$ is unbounded.

(ii) The optimal value of $\mathrm{D}(\mathcal{I}, \mathcal{J})$ is greater than the objective value of the incumbent solution, i.e., the best feasible solution of $(\mathrm{P})$ found so far.

(iii) There is no duality gap between $\mathrm{P}(\mathcal{I}, \mathcal{J})$ and $\mathrm{D}(\mathcal{I}, \mathcal{J})$. 
We now give more details about the bounding operations based on the above three conditions.

If the dual problem $\mathrm{D}(\mathcal{I}, \mathcal{J})$ is unbounded, then the primal problem $\mathrm{P}(\mathcal{I}, \mathcal{J})$ is infeasible from the weak duality theorem. In this case, any subproblem generated from the current subproblem by restricting the sign of some of its variables cannot be feasible. Hence we can discard the current subproblem $\mathrm{P}(\mathcal{I}, \mathcal{J})$.

If the optimal value of $\mathrm{D}(\mathcal{I}, \mathcal{J})$, which is a lower bound of the optimal value of $\mathrm{P}(\mathcal{I}, \mathcal{J})$ by Theorem 2.1, is greater than the objective value of the incumbent solution, we have no chance to obtain an optimal solution of $(\mathrm{P})$ by generating subproblems from $\mathrm{P}(\mathcal{I}, \mathcal{J})$ further. So we can discard the current subproblem.

If we find out that there is no duality gap between $\mathrm{P}(\mathcal{I}, \mathcal{J})$ and $\mathrm{D}(\mathcal{I}, \mathcal{J})$, then this means the subproblem $\mathrm{P}(\mathcal{I}, \mathcal{J})$ is just solved. For this reason, we need not generate new subproblems from $\mathrm{P}(\mathcal{I}, \mathcal{J})$ further, and we can discard the current subproblem. Moreover, if the optimal solution of $\mathrm{P}(\mathcal{I}, \mathcal{J})$ is better than the incumbent solution, then we replace the incumbent solution by the optimal solution of $\mathrm{P}(\mathcal{I}, \mathcal{J})$. We may check if there is no duality gap between $\mathrm{P}(\mathcal{I}, \mathcal{J})$ and $\mathrm{D}(\mathcal{I}, \mathcal{J})$ by solving the following system of absolute value equations and inequalities:

$$
\begin{aligned}
c^{\top} x+d^{\top}|x| & =f_{D}^{*}, \\
A x+B|x| & =b, \\
\tilde{H} x+\tilde{K}|x| & \geq \tilde{p},
\end{aligned}
$$

where $f_{D}^{*}$ is the optimal objective value of the dual problem $\mathrm{D}(\mathcal{I}, \mathcal{J})$. If the system (S1) has a solution, then $\mathrm{P}(\mathcal{I}, \mathcal{J})$ and $\mathrm{D}(\mathcal{I}, \mathcal{J})$ have no duality gap. Moreover, by Theorem 2.2, it is an optimal solution of $\mathrm{P}(\mathcal{I}, \mathcal{J})$.

We now formally state the algorithm.

\section{Branch-and-Bound Algorithm for AVP:}

- Step 0. Let $\mathcal{I}:=\emptyset$ and $\mathcal{J}:=\emptyset$. Find a feasible solution of problem $(P)=$ $\mathrm{P}(\emptyset, \emptyset)$. Let it be the incumbent solution and let $f^{*}$ be the objective value at the incumbent solution. Set $\mathcal{A}:=\{\mathrm{P}(\emptyset, \emptyset)\}$.

- Step 1. Choose a subproblem $\mathrm{P}(\mathcal{I}, \mathcal{J})$ from the set $\mathcal{A}$.

- Step 1-a. If the dual problem $\mathrm{D}(\mathcal{I}, \mathcal{J})$ of $\mathrm{P}(\mathcal{I}, \mathcal{J})$ is infeasible, then go to Step 2. If $\mathrm{D}(\mathcal{I}, \mathcal{J})$ is unbounded, then fathom $\mathrm{P}(\mathcal{I}, \mathcal{J})$. Set $\mathcal{A}:=\mathcal{A} \backslash\{\mathrm{P}(\mathcal{I}, \mathcal{J})\}$ and go to Step 3.

- Step 1-b. Let $f_{D}^{*}$ be the optimal objective value of the dual problem $\mathrm{D}(\mathcal{I}, \mathcal{J})$. If it satisfies $f_{D}^{*}>f^{*}$, then fathom $\mathrm{P}(\mathcal{I}, \mathcal{J})$. Set $\mathcal{A}:=\mathcal{A} \backslash\{\mathrm{P}(\mathcal{I}, \mathcal{J})\}$ and go to Step 3.

- Step 1-c. Solve the system (S1) of absolute value equations and inequalities. If we fail to get a solution of (S1), then go to Step 2. If we get a solution of (S1) and, in addition, the objective value at the solution, denoted $f_{(\mathcal{I}, \mathcal{J})}$, satisfies $f_{(\mathcal{I}, \mathcal{J})} \geq f^{*}$, then $\mathrm{P}(\mathcal{I}, \mathcal{J})$ is fathomed immediately. If $f_{(\mathcal{I}, \mathcal{J})}<f^{*}$, then set $f^{*}:=f_{(\mathcal{I}, \mathcal{J})}$, update the incumbent solution, and fathom $\mathrm{P}(\mathcal{I}, \mathcal{J})$. Set $\mathcal{A}:=\mathcal{A} \backslash\{\mathrm{P}(\mathcal{I}, \mathcal{J})\}$ and go to Step 3 .

- Step 2. Choose a variable $x_{i}$ such that $i \notin \mathcal{I} \cup \mathcal{J}$ as the branching variable, and generate two subproblems $\mathrm{P}(\mathcal{I} \cup\{i\}, \mathcal{J})$ and $\mathrm{P}(\mathcal{I}, \mathcal{J} \cup\{i\})$ from $\mathrm{P}(\mathcal{I}, \mathcal{J})$. Set $\mathcal{A}:=\mathcal{A} \cup\{\mathrm{P}(\mathcal{I} \cup\{i\}, \mathcal{J}), \mathrm{P}(\mathcal{I}, \mathcal{J} \cup\{i\})\} \backslash\{\mathrm{P}(\mathcal{I}, \mathcal{J})\}$, and return to Step 1.

- Step 3. If $\mathcal{A}=\emptyset$, then terminate. The incumbent solution is an optimal solution of the original problem $(\mathrm{P})$. Otherwise, return to Step 1.

In order to get a feasible solution of (P) in Step 0 and to solve (S1) in Step 1-c, we can use the successive linearization algorithm (SLA) for the system of absolute 
value equations and inequalities. This algorithm was first proposed by Mangasarian [10] to solve AVE. We extend the algorithm so as to deal with a system that also contains absolute value inequalities (AVIs).

Here we describe the SLA for the system

$$
\left\{\begin{array}{l}
A x+B|x|=b \\
H x+K|x| \geq p
\end{array}\right.
$$

which represents the constraints of $(\mathrm{P})$. The algorithm can similarly be applied to solve the system (S1).

First we give a result that relates the AVE-AVI system (S2) to the following concave minimization problem constructed from (S2):

$$
\begin{array}{r}
\min _{\left(x, t, s_{1}, s_{2}\right) \in \mathbb{R}^{n+n+m+\ell}} \epsilon\left(-e^{\top}|x|+e^{\top} t\right)+e^{\top} s_{1}+e^{\top} s_{2} \\
\text { s.t. }-s_{1} \leq A x+B t-b \leq s_{1}, \\
-H x-K t+p \leq s_{2}, \\
0 \leq s_{2}, \\
-t \leq x \leq t,
\end{array}
$$

where $\epsilon>0$ and $e$ is the vector of ones.

Proposition 3.1: If (S2) is solvable, then there exists some $\bar{\epsilon}>0$ such that, for any $\epsilon \in(0, \bar{\epsilon}]$, any solution $\left(\bar{x}, \bar{t}, \overline{s_{1}}, \overline{s_{2}}\right)$ of (1) satisfies

$$
\begin{aligned}
|\bar{x}| & =\bar{t}, \\
A \bar{x}+B|\bar{x}| & =b, \\
H \bar{x}+K|\bar{x}| & \geq p .
\end{aligned}
$$

Proof: The proof is analogous to that of Proposition 3 in [10].

From this result, a solution of the AVE-AVI system (S2) may be obtained by solving the concave minimization problem (1) with a sufficiently small $\epsilon>0$. We now give the SLA for the AVE-AVI system (S2), which is an extension of the SLA for AVE [10]. Let $z=\left(x, t, s_{1}, s_{2}\right)^{\top}$. Denote the feasible region of problem (1) by $Z$ and its objective function by $\theta(z)$.

\section{SLA for AVE-AVI:}

- Step 0. Choose a starting point $z^{0} \in Z$. Set $k:=0$.

- Step 1. Given $z^{k}$, find $z^{k+1}$ such that

$$
z^{k+1} \in \arg \operatorname{vertex} \min _{z \in Z}\left(\xi^{k}\right)^{\top}\left(z-z^{k}\right)
$$

where $\xi^{k}$ is a subgradient of $\theta(z)$ at $z^{k}$, and $\arg$ vertex $\min _{z \in Z}\left(\xi^{k}\right)^{\top}\left(z-z^{k}\right)$ is the set of vertex solutions of the linear program: $\min _{z \in Z}\left(\xi^{k}\right)^{\top}\left(z-z^{k}\right)$.

- Step 2. If $\left(\xi^{k}\right)^{\top}\left(z^{k+1}-z^{k}\right)=0$, then stop. Otherwise, return to Step 1 with $k$ increased by one.

In our numerical experiments, we compute a subgradient $\xi^{k}$ of $\theta(z)$ at $z^{k}$ as 
follows:

$$
\xi^{k}=\left(\begin{array}{c}
-\epsilon g^{k} \\
\epsilon e \\
e \\
e
\end{array}\right) \in \mathbb{R}^{n+n+m+\ell} \quad \text { with } \quad g_{i}^{k}=\left\{\begin{array}{ll}
1 & \left(x_{i}^{k}>0\right) \\
0 & \left(x_{i}^{k}=0\right) \\
-1 & \left(x_{i}^{k}<0\right)
\end{array}, \quad i=1, \cdots, n .\right.
$$

As is well-known, a concave minimization problem has at least one optimal solution at a vertex of the feasible region, provided a solution exists. Taking this fact into account, the SLA tries to find an optimal solution of (1) by solving a sequence of linear programs formed by linearizing the objective function of problem (1). The sequence generated by the SLA finitely converges to a point that satisfies a necessary optimality condition for the concave minimization problem $[8,10]$. Notice that the solution obtained by this algorithm is not guaranteed to be a global optimal solution of (1). Nevertheless, we can easily check if the computed solution actually satisfies (S2) by direct substitution.

We now show the way to generate subproblems in Step 2 of the branch-andbound algorithm. Recall that Step 2 is visited after either of the following two cases occurs.

Case 1. In Step 1-a, $\mathrm{D}(\mathcal{I}, \mathcal{J})$ is infeasible.

Case 2. In Step 1-c, (S2) cannot be solved.

If Case 1 occurs, then we generate two subproblems by choosing any variable $x_{i}$ such that $i \notin \mathcal{I} \cup \mathcal{J}$ as the branching variable. In Case 2, we fail to have a solution of (S2), but a local optimal solution of problem (1) is obtained. In this case, we choose as the branching variable a variable $x_{i}(i \notin \mathcal{I} \cup \mathcal{J})$ such that $\left|x_{i}\right| \geq\left|x_{j}\right|$ for all $j \notin \mathcal{I} \cup \mathcal{J}$ at the obtained local optimal solution of (1).

In Step 1, a certain rule should be used to choose an active subproblem $\mathrm{P}(\mathcal{I}, \mathcal{J}) \in$ $\mathcal{A}$. In the numerical experiments reported in the next section, we use the depth-first search, which generally chooses an active subproblem corresponding to the farthest node from the root node in the enumeration tree. In particular, when we return to Step 1 after generating two subproblems, we choose one of these subproblems. In this case, the choice depends on the above-mentioned two cases. If we generate two subproblems in Step 2 after Case 1 occurs, then we choose any of the two subproblems. In Case 2, as we mentioned above, we have a local optimal solution of (1) at hand. In this case, if the branching variable $x_{i}$ in the local optimal solution takes a positive value, then we choose subproblem $\mathrm{P}(\mathcal{I} \cup\{i\}, \mathcal{J})$. Otherwise, we choose $\mathrm{P}(\mathcal{I}, \mathcal{J} \cup\{i\})$.

\section{Numerical Experiments}

In this section, we consider facility location problems (FLPs) as an application of AVP, and show some numerical results with the proposed branch-and-bound algorithm applied to some examples of FLPs. All computations were carried out on an Intel ${ }^{\mathrm{R}}$ Core $^{\mathrm{TM}} 2$ Duo $3 \mathrm{GHz}$ machine with a MATLAB code. The CPLEX was used to solve linear programs in the SLA.

FLP is the problem of finding optimal locations of facilities in a given area, and it can be formulated as mathematical programs of different natures depending on the type of constraints and optimization criteria [2]. Generally speaking, there are two kinds of facilities from the residents' standpoint. The first category is a desirable facility such as schools, libraries and fire stations. Such a facility should be located as closely as possible to the residents. The other category is an undesirable facility, 
which includes incineration plants, electric power stations, chemical factories and so on. These facilities should be located far from the residential area. From the viewpoint of geography, there are three kinds of areas in which facilities can be located, i.e., continuous spaces, discrete spaces, and networks. Furthermore, the distance between two facilities or between a facility and a residential district can be measured by using various norms such as the Euclidean, $\ell_{1}$, and $\ell_{\infty}$ norms.

In our numerical experiments, we consider two types of FLPs on a continuous space with the $\ell_{1}$ distance, which can be reformulated as AVPs. Note that the $\ell_{1}$ distance between two points $x$ and $y$ can be represented as $e^{\top}|x-y|$.

\subsection{Minimax Location Problem}

A minimax multi-facility location problem can be formulated as follows [2]:

$$
\begin{aligned}
& \min \max \left\{\max _{i \in I, j \in J} \alpha_{i j} e^{\top}\left|x^{i}-P^{j}\right|, \max _{\substack{i, k \in I, i \neq k \\
(i \in I),}} \beta_{i k} e^{\top}\left|x^{i}-x^{k}\right|\right\} \\
& \text { s.t. } \\
& \qquad x^{i} \in X \quad(i \in I),
\end{aligned}
$$

where $x^{i} \in \mathbb{R}^{2}(i \in I)$ and $P^{j} \in \mathbb{R}^{2}(j \in J)$ denote the locations of the new and the existing facilities, respectively, $I$ and $J$ are finite index sets, $\alpha_{i j}$ and $\beta_{i k}$ are positive weighting factors, and $X \subset \mathbb{R}^{2}$ is the region in which the facilities are located.

The problem is to minimize the maximum weighted distance between new and existing facilities, and between new facilities themselves. If each existing facility is regarded as a residential district, then this problem represents a mathematical model of locating desirable facilities, such as schools and fire stations, in a city. This kind of problems has been well-studied for the past decades. In particular, using the $\ell_{1}$ norm as the distance function, Konforty and Tamir [6] studied the minimax single facility location problem with a forbidden region around each existing facility.

Problem (2) can be rewritten as the following problem by introducing a new variable $z \in \mathbb{R}$ :

$$
\begin{array}{rl}
\left(\mathrm{P}_{\mathrm{a}}\right) \min _{x, z} & z \\
\mathrm{s.t.} \quad & z \geq \alpha_{i j} e^{\top}\left|x^{i}-P^{j}\right|(i \in I, j \in J), \\
& z \geq \beta_{i k} e^{\top}\left|x^{i}-x^{k}\right|(i, k \in I, i \neq k), \\
& x^{i} \in X \quad(i \in I) .
\end{array}
$$

If $X$ is a convex polyhedron, $\left(\mathrm{P}_{\mathrm{a}}\right)$ is easy to solve because it reduces to a linear program. Here, we deal with the more general case where $X$ is a nonconvex region.

We now give the detail of the problem that we solve in numerical experiments. We define the region $X$ as the set of points $x=\left(x_{1}, x_{2}\right)^{\top} \in \mathbb{R}^{2}$ that satisfy the following inequalities:

$$
\begin{aligned}
& \left|x_{1}\right|+\left|x_{2}\right|+0.2 x_{1}+0.4 x_{2} \leq 10 \\
& \left|x_{1}\right|+2\left|x_{2}+12\right|-0.5 x_{1} \geq 12 \\
& \left|x_{1}+2\right|+1.5\left|x_{2}+1\right|-0.3 x_{1}-0.5 x_{2} \geq 5
\end{aligned}
$$

The region $X$ is nonconvex, as shown in Fig. 2. Notice that since the region $X$ is described by (3), problem $\left(\mathrm{P}_{\mathrm{a}}\right)$ is an instance of AVP.

In the numerical experiments, we let $I=\{1,2\}, J=\{1,2,3\}$ and set the locations of the existing facilities as $P^{1}=(-7,-5), P^{2}=(-2,5), P^{3}=(7,-1)$. Moreover, we choose the positive weight $\beta_{12}=1.0$, and use two data sets for the weights $\alpha_{i j}$ given as follows: 


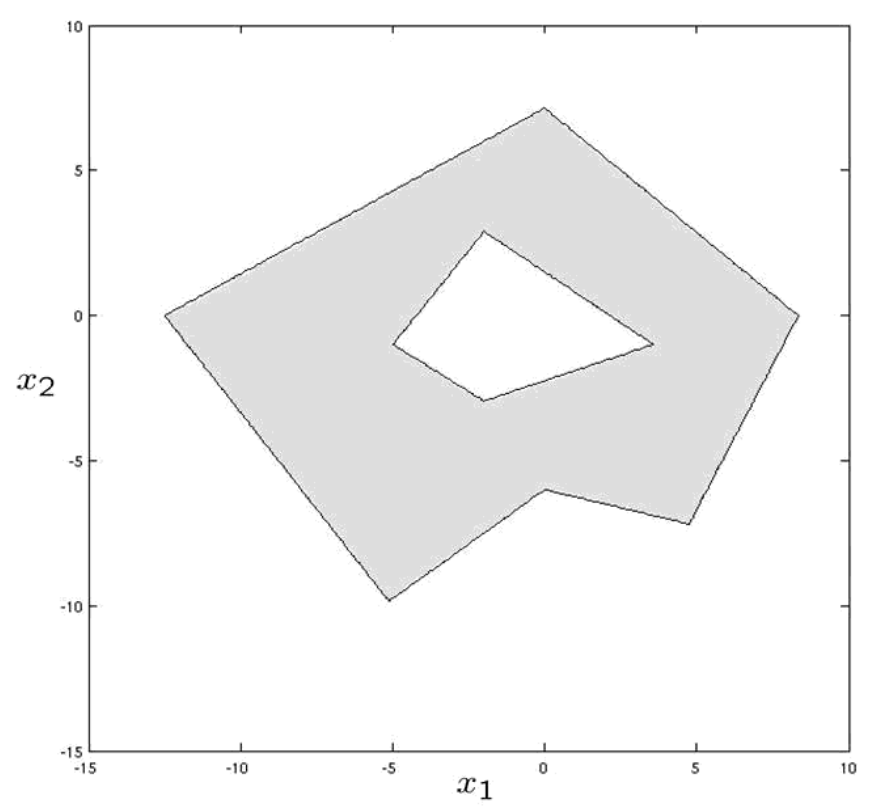

Figure 2. Region $X$ where the facilities are located.

$$
\left(\alpha_{11}, \alpha_{12}, \alpha_{13}, \alpha_{21}, \alpha_{22}, \alpha_{23}\right)=(0.5,1.0,0.7,0.5,0.7,1.0)
$$

and

$$
\left(\alpha_{11}, \alpha_{12}, \alpha_{13}, \alpha_{21}, \alpha_{22}, \alpha_{23}\right)=(0.7,1.0,0.5,0.5,1.0,0.7)
$$

The problems with $\alpha_{i j}$ 's given by (4) and (5) are called Minimax-1 and Minimax-2, respectively. The branch-and-bound method was able to find solutions of Minimax1 and Minimax-2, which are given by $x^{1}=(0,1.5), x^{2}=(0.98,-1.9)$ and $x^{1}=$ $(-3.42,1.05), x^{2}=(-0.07,1.55)$, respectively. The solutions are depicted in Fig. 3 and Fig. 4. For each problem, the CPU time, the numbers of subproblems fathomed in Step 1-a, Step 1-b, Step 1-c, and the number of nodes explored are summarized in Table 1.

Table 1: Results for minimax location problems

\begin{tabular}{cccccc}
\hline & CPU time (sec) & Step 1-a & Step 1-b & Step 1-c & No. of nodes explored \\
\hline Minimax-1 & 0.55 & 16 & 63 & 4 & 164 \\
Minimax-2 & 2.7 & 230 & 96 & 30 & 710 \\
\hline
\end{tabular}

\subsection{Maximin Location Problem}

A maximin multi-facility location problem is generally formulated as follows [2]:

$$
\begin{array}{lc}
\max \min & \left\{\min _{i \in I, j \in J} \alpha_{i j} e^{\top}\left|x^{i}-P^{j}\right|, \min _{i, k \in I, i \neq k} \beta_{i k} e^{\top}\left|x^{i}-x^{k}\right|\right\} \\
\text { s.t. } & x^{i} \in X \quad(i \in I),
\end{array}
$$




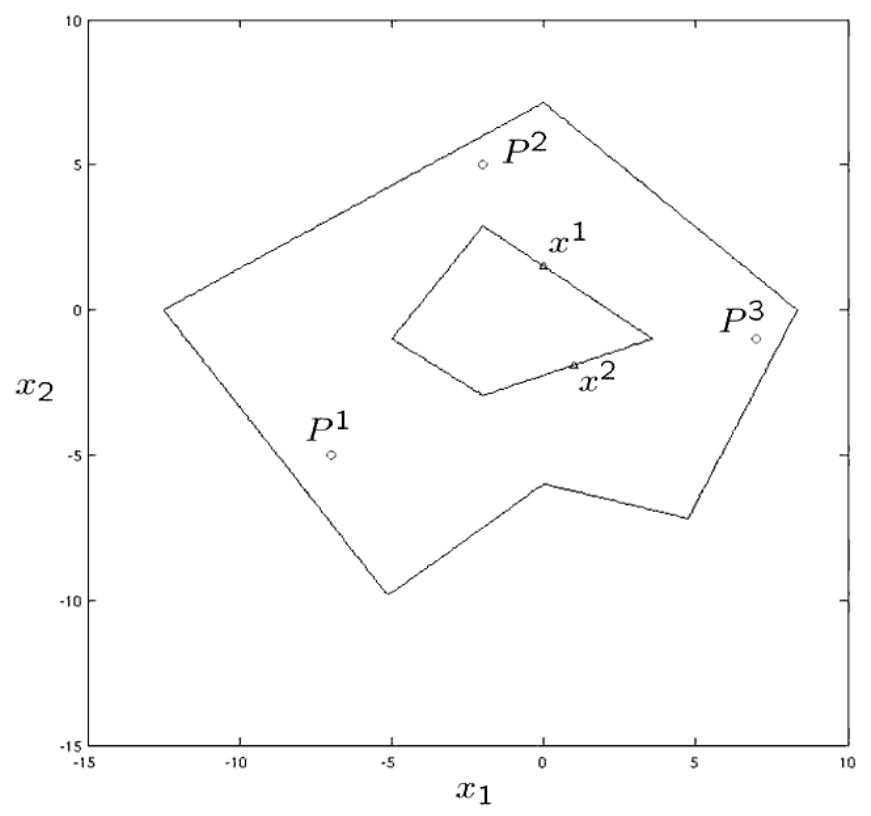

Figure 3. Solution of Minimax-1

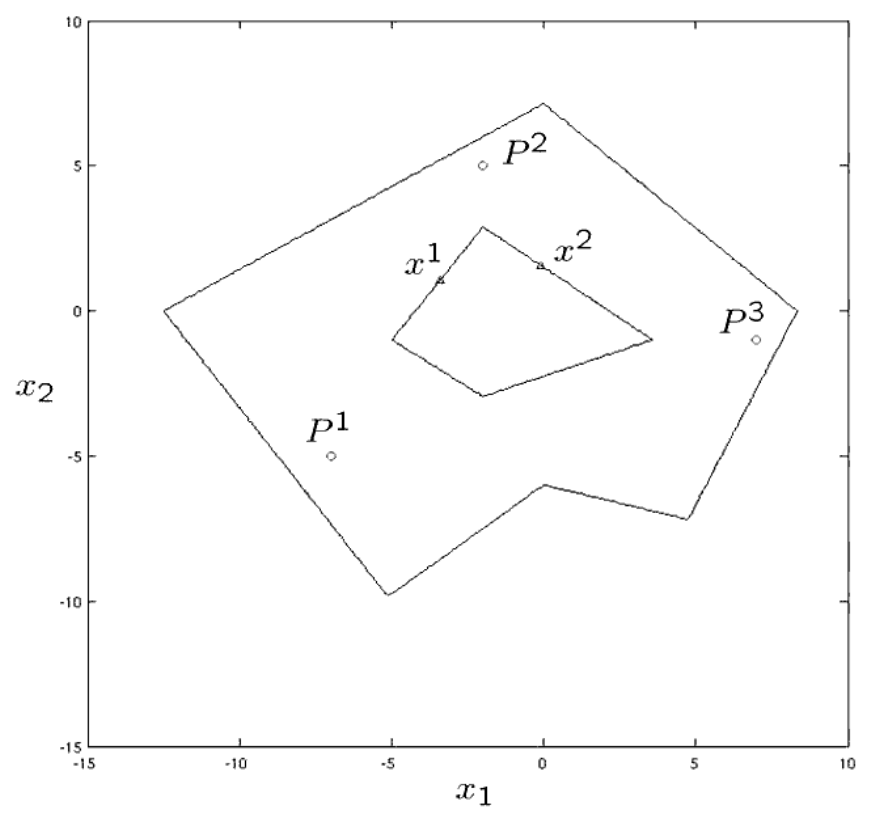

Figure 4. Solution of Minimax-2

where $x^{i}, P^{j}, \alpha_{i j}, \beta_{i j}$ and $X$ represent the same stuffs as in problem (2). Unlike the minimax location problem, this problem maximizes the minimum weighted distances between new and existing facilities, and between new facilities themselves. The maximin location problem will be useful in locating competing facilities such as convenience stores and gas stations.

Sayin [17] and Nadirler and Karasakal [13] reformulated single facility maximin location problem on a convex region with the $\ell_{1}$ distance as a mixed integer pro- 
gram. Tamir [18] proposed an algorithm for two-facility maximin location problems on a convex region with the $\ell_{1}$ distance. In these approaches, the region for locating facilities is assumed to be convex. Here we solve multi-facility location problems on a nonconvex region.

Problem (6) can be rewritten as the following problem by introducing a new variable $z \in \mathbb{R}[18,19]$ :

$$
\begin{array}{cl}
\left(\mathrm{P}_{\mathrm{b}}\right) \max _{x, z} & z \\
\text { s.t. } \quad & z \leq \alpha_{i j} e^{\top}\left|x^{i}-P^{j}\right|(i \in I, j \in J), \\
& z \leq \beta_{i k} e^{\top}\left|x^{i}-x^{k}\right|(i, k \in I, i \neq k), \\
& x^{i} \in X \quad(i \in I) .
\end{array}
$$

Notice that, unlike the inequality constraints in $\left(\mathrm{P}_{\mathrm{a}}\right)$, those in this problem are nonconvex.

In the numerical experiments, we let the index sets of the new and the existing facilities be $I=\{1,2\}$ and $J=\{1,2,3\}$, respectively. In addition, we set all the positive weights $\alpha_{i j}$ and $\beta_{12}$ to be 1 . The region $X$ is the nonconvex region described by (3). Moreover, the locations of the existing facilities are given in the following two data sets:

$$
P^{1}=(-10,-1), P^{2}=(-5,2), P^{3}=(2,4)
$$

and

$$
P^{1}=(-9,1), P^{2}=(-1,-3), P^{3}=(6,-2) .
$$

The problems with the data sets (7) and (8) are called Maximin-1 and Maximin2 , respectively. By using the proposed branch-and-bound method, we obtained a solution $x^{1}=(-2.42,-7.81), x^{2}=(6.27,-4.12)$ for Maximin-1 and a solution $x^{1}=(-5.12,-9.84), x^{2}=(0,6.96)$ for Maximin-2. Those solutions are shown in Fig. 5 and Fig. 6. For each problem, the CPU time, the numbers of subproblems fathomed in Step 1-a, Step 1-b, Step 1-c, and the number of nodes explored are shown in Table 2.

Table 2: Results for maximin location problems

\begin{tabular}{lccccc}
\hline & CPU time (sec) & Step 1-a & Step 1-b & Step 1-c & No. of nodes explored \\
\hline Maximin-1 & 7.9 & 753 & 492 & 13 & 2514 \\
Maximin-2 & 14.1 & 1478 & 686 & 18 & 4362 \\
\hline
\end{tabular}

For each of the above examples Minimax-1, 2, and Maximin-1, 2, the AVP in the form of $(\mathrm{P})$ has 27 variables and 22 constraints. From the results shown in this section, we observe that a global optimal solution of each problem was found by exploring only a small number of nodes compared with the number of all possible nodes $\left(2^{27}-1\right)$ in the enumeration tree. Although $\left(\mathrm{P}_{\mathrm{a}}\right)$ and $\left(\mathrm{P}_{\mathrm{b}}\right)$ have the same numbers of variables and constraints, there is a significant difference in the CPU time between these two types of problems, as shown in Table 1 and Table 2 . The reason for this phenomenon may be explained as follows. The minimax location problem (2) has a convex objective function, although the feasible region is nonconvex. On the other hand, the objective function of the maximin location problem (6) is neither convex nor concave. Such a problem is considered to be much more difficult to deal with in practice. 


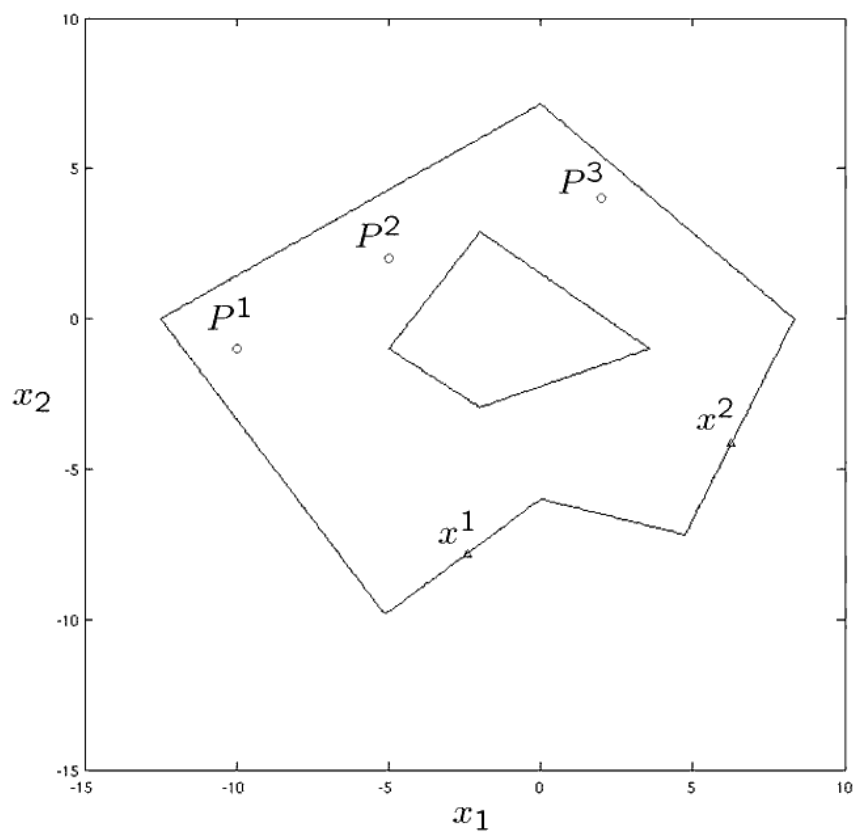

Figure 5. Solution of Maximin-1

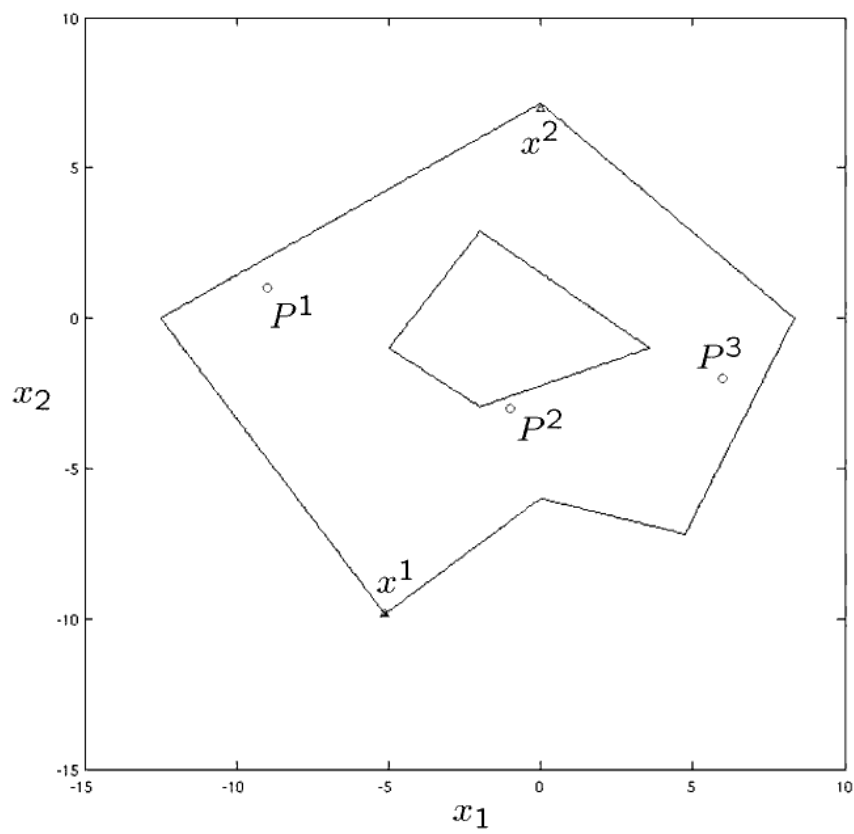

Figure 6. Solution of Maximin-2

\section{Conclusion}

In this paper, we have proposed an algorithm for the AVP, which is based on the branch-and-bound method. We have also carried out numerical experiments for nonconvex multi-facility location problems with the $\ell_{1}$ norm, which can naturally 
be reformulated as AVPs. The numerical results demonstrate the validity of the proposed algorithm.

\section{Acknowledgement}

This work was supported in part by a Grant-in-Aid for Scientific Research from Japan Society for the Promotion of Science.

\section{References}

[1] L. Caccetta, B. Qu and G. Zhou, A globally and quadratically convergent method for absolute value equations. Comput. Optim. Appl. 48 (2011), pp. 45-58.

[2] R.Z. Farahani and M. Hekmatfar, eds., Facility Location: Concepts, Models, Algorithms and Case Studies, Springer Verlag, Berlin/Heidelberg, 2009.

[3] R. Horst, P.M. Pardalos and N.V. Thoai, Introduction to Global Optimization, Kluwer, Dordrechts, 2000.

[4] S.L. Hu, Z.J. Huang and Q. Zhang, A generalized Newton method for absolute value equations associated with second order cones, J. Comput. Appl. Math. 235 (2011), pp. 1490-1501.

[5] T. Ibaraki, Enumerative Approaches to Combinatorial Optimization, Ann. Oper. Res. 10 \& 11 , J.C. Baltzer, Basel, 1987.

[6] Y. Konforty and A. Tamir, The single facility location problem with minimum distance constraints, Location Sci. 5 (1987), pp. 147-163.

[7] Z.Q. Luo, J.S. Pang and D. Ralph, Mathematical Programs with Equilibrium Constraints, Cambridge University Press, Cambridge, 1996.

[8] O.J. Mangasarian, Solution of general linear complementarity problems via nondifferentiable concave minimization, Acta Math. Vietnamica 22 (1997), pp. 199-205.

[9] O.L. Mangasarian, Absolute value equation solution via concave minimization, Optim. Lett. 1 (2007), pp. $3-8$.

[10] O.L. Mangasarian, Absolute value programming, Comput. Optim. Appl. 36 (2007), pp. 43-53.

[11] O.L. Mangasarian, A generalized Newton method for absolute value equations, Optim. Lett. 3 (2009), pp. 101-108.

[12] O.L. Mangasarian and R.R. Meyer, Absolute value equations, Linear Algebra Appl. 419 (2006), pp. 359-367.

[13] D. Nadirler and E. Karasakal, Mixed integer programming-based solution procedure for single-facility location with maximin of rectilinear distance, J. Oper. Res. Soc. 59 (2008), pp. 563-570.

[14] O. Prokopyev, On equivalent reformulations for absolute value equations, Comput. Optim. Appl. 44 (2009), pp. 363-372.

[15] J. Rohn, A theorem of the alternatives for the equation $A x+B|x|=b$, Linear Multilinear A. 52 (2004), pp. 421-426.

[16] J. Rohn, On unique solvability of the absolute value equation, Optim. Lett. 3 (2009), pp. 603-606.

[17] S. Sayin, A mixed integer programming formulation for the 1-maximin problem, J. Oper. Res. Soc. 51 (2000), pp. 371-375.

[18] A. Tamir, Locating two obnoxious facilities using the weighted maximin criterion, Oper. Res. Lett. 34 (2006), pp. 97-105.

[19] S.B. Welch, S. Salhi and Z. Drezner, The multifacility maximin planar location problem with facility interaction, IMA J. Manag. Math. 17 (2006), pp. 397-412.

[20] C. Zhang and Q.J. Wei, Global and finite convergence of a generalized Newton method for absolute value equations, J. Optim. Theory Appl. 143 (2009), pp. 391-403. 